\title{
BMJ
}

\section{Efficacy of standardised manual therapy and home exercise programme for chronic rotator cuff disease: randomised placebo controlled trial}

\author{
Kim Bennell, professor, ${ }^{1}$ Elin Wee, physiotherapist and research assistant, ${ }^{1}$ Sally Coburn, physiotherapist, ${ }^{1,2}$ \\ Sally Green, professor, ${ }^{3}$ Anthony Harris, professor, ${ }^{4}$ Margaret Staples, biostatistician, ${ }^{5,6}$ Andrew Forbes, \\ professor, ${ }^{6}$ Rachelle Buchbinder, professor ${ }^{5,6}$
}

${ }^{1}$ Centre for Health, Exercise and Sports Medicine, Department of Physiotherapy, School of Health Sciences, University of Melbourne, Parkville 3010, Victoria, Australia

${ }^{2}$ Department of Physiotherapy, Royal Melbourne Hospital, Melbourne

${ }^{3}$ Monash Institute of Health Services Research, Monash University, Melbourne

${ }^{4}$ Centre for Health Economics, Monash University

${ }^{5}$ Monash Department of Clinical Epidemiology, Cabrini Hospital, Melbourne

${ }^{6}$ Department of Epidemiology and Preventive Medicine, School of Public Health and Preventive Medicine, Monash University

Correspondence to: $\mathrm{K}$ Bennell k.bennell@unimelb.edu.au

Cite this as: BMJ 2010;340:c2756 doi:10.1136/bmj.c2756

\section{ABSTRACT}

Objective To investigate the efficacy of a programme of manual therapy and exercise treatment compared with placebo treatment delivered by physiotherapists for people with chronic rotator cuff disease.

Design Randomised, participant and single assessor blinded, placebo controlled trial.

Setting Metropolitan region of Melbourne, Victoria, Australia.

Participants 120 participants with chronic ( $>3$ months) rotator cuff disease recruited through medical practitioners and from the community.

Interventions The active treatment comprised a manual therapy and home exercise programme; the placebo treatment comprised inactive ultrasound therapy and application of an inert gel. Participants in both groups received 10 sessions of individual standardised treatment over 10 weeks. For the following 12 weeks, the active group continued the home exercise programme and the placebo group received no treatment.

Main outcome measures The primary outcomes were pain and function measured by the shoulder pain and disability index, average pain on movement measured on an 11 point numerical rating scale, and participants' perceived global rating of overall change.

Results 112 (93\%) participants completed the 22 week trial. At 11 weeks no difference was found between groups for change in shoulder pain and disability index (3.6, $95 \%$ confidence interval -2.1 to 9.4$)$ or change in pain (0.7, -0.1 to 1.5$)$; both groups showed significant improvements. More participants in the active group reported a successful outcome (defined as "much better"), although the difference was not statistically significant: $42 \%(24 / 57)$ of active participants and $30 \%$ (18/61) of placebo participants (relative risk 1.43, 0.87 to 2.34). The active group showed a significantly greater improvement in shoulder pain and disability index than did the placebo group at 22 weeks (between group difference $7.1,0.3$ to 13.9), although no significant difference existed between groups for change in pain $(0.9,-0.03$ to 1.7$)$ or for the percentage of participants reporting a successful treatment outcome (relative risk
$1.39,0.94$ to 2.03$)$. Several secondary outcomes favoured the active group, including shoulder pain and disability index function score, muscle strength, interference with activity, and quality of life.

Conclusion A standardised programme of manual therapy and home exercise did not confer additional immediate benefits for pain and function compared with a realistic placebo treatment that controlled for therapists' contact in middle aged to older adults with chronic rotator cuff disease. However, greater improvements were apparent at follow-up, particularly in shoulder function and strength, suggesting that benefits with active treatment take longer to manifest.

Trial registration Clinical trials NCT00415441.

\section{INTRODUCTION}

Shoulder disorders are a common cause of persistent musculoskeletal morbidity, ${ }^{12}$ particularly in the middle to older age groups. ${ }^{3}$ Pain and compromised shoulder function have a substantial impact on tasks essential to daily living, as well as on sleep. ${ }^{4}$ Shoulder disorders are a common reason for seeking medical care and may require surgical intervention in up to $28 \%$ of cases. ${ }^{5-7}$ Shoulder disorders can thus lead to considerable disability, reduced health related quality of life, absenteeism from work, and use of healthcare resources. ${ }^{489}$

Although definitions of different diagnostic categories of shoulder pain are controversial, a large proportion of shoulder problems can be classified as "rotator cuff disease," the most common cause of shoulder pain in primary care. ${ }^{10}$ The term, or its variants such as impingement syndrome, may include a spectrum of pathologies of rotator cuff disease (such as subacromial bursitis, partial rotator cuff tears, and bicipital tendinosis), but they are characterised clinically by pain with abduction (painful arc) and signs of impingement. ${ }^{11}$ Although standard criteria have not been established for use in clinical trials, most trials that have assessed interventions for rotator cuff disease have used variations of these features to select their study populations. ${ }^{12-14}$ Rotator cuff disease differs 
from other major diagnostic categories of shoulder pain such as adhesive capsulitis, osteoarthritis, and calcific tendinitis, which are known to have different presentations, underlying causes, prognoses, and responses to treatment.

A combination of modalities of physiotherapy, such as manual therapy and exercise, is often used in the management of rotator cuff disease. ${ }^{15}$ These aim to correct modifiable physical impairments thought to contribute to pain and dysfunction rather than to treat the specific pathology. These impairments include rotator cuff and scapular muscle weakness and dysfunction, tightness of the posterior capsule and other soft tissues, and postural abnormalities. ${ }^{16}$ Little conclusive evidence supports or refutes the efficacy of different physiotherapy programmes given the variable methodological quality of the trials, including a lack of placebo control and the fact that many tested a single modality despite multimodality treatment being the most common way in which physiotherapists treat shoulder disorders. ${ }^{1317-20}$ The conclusions and recommendations of recent systematic reviews support the need for further clinical trials. ${ }^{21-23}$ The primary aim of this trial was, therefore, to determine whether a 10 week programme of standardised manual therapy and home exercise delivered by a physiotherapist improves shoulder pain and function more than placebo treatment does in people with chronic rotator cuff disease.

\section{METHODS}

\section{Participants}

Between March 2004 and November 2007, we recruited people with chronic rotator cuff disease through medical practitioners and from the community through print and radio media. We required all participants to have a plain radiograph of the shoulder to check for exclusions (see below), and we required potential participants recruited directly from the community to have the diagnosis of rotator cuff disease confirmed by a medical practitioner. After an initial screen by telephone, an experienced physiotherapist (EW or SC) did a physical examination. Inclusion criteria were age over 18 years, shoulder pain for more than three months, severity of pain on movement rated greater than $3 / 10$ on an $0-10$ numerical rating scale, pain on active abduction or external rotation, and a positive quick test for shoulder impingement. ${ }^{24}$ Exclusion criteria were resting severity of shoulder pain greater than $7 / 10$; reason to suspect a complete rotator cuff tear (for example, substantial shoulder weakness, a positive drop-arm sign, or a high riding humerus on plain radiograph); previous shoulder surgery; radiological evidence of shoulder osteoarthritis, calcification, or previous fracture; systemic pathology including inflammatory joint disease or neoplastic disorders; more than $50 \%$ restriction of passive range of motion in two or more planes; shoulder pain referred from vertebral structures diagnosed by spinal clearing test $^{25}$; symptoms of complex regional pain syndrome; active intervention in the previous three months, including corticosteroid injection, arthrographic distension of the glenohumeral joint with corticosteroid and saline (hydrodilatation), or physiotherapy; antiinflammatory drugs in the previous two weeks; and inability to understand written and spoken English.

\section{Procedures}

We did a randomised, participant and assessor blinded, controlled trial. Participants had a baseline assessment and were randomised in permuted blocks of six and eight, stratified by treating physiotherapist, to receive either active manual therapy and home exercise treatment or placebo treatment according to a computer generated table of random numbers created by the study biostatistician (AF). Allocations were sealed in opaque and consecutively numbered envelopes kept in a central locked location. An independent administrator opened the envelopes in sequence and then revealed the group allocation to the relevant physiotherapist by facsimile just before the participant presented for treatment.

\section{Interventions}

Details about the interventions have been published previously. ${ }^{26}$ Fourteen musculoskeletal physiotherapists (all with more than four years of relevant clinical experience) from 12 centres (two public hospital physiotherapy departments and 10 private physiotherapy clinics) were trained to provide both interventions. Therapists attended initial training sessions and were given a detailed treatment manual. We could not blind the therapists to treatment group. Both interventions were standardised and comprised individual sessions twice weekly for the first fortnight, once a week for the next four weeks, then once a fortnight in the last four weeks (10 visits, 30-45 minutes each). To minimise the risk of participants meeting, appointments were scheduled at different times. We assessed therapists' adherence to the protocol by completion of a treatment log. Simple analgesia was permitted, but participants were asked to refrain from seeking other forms of treatment during the trial. Treatment in both groups was provided at no cost to the participant.

We based the active intervention on the literature and on the results of a formal written survey of 16 Australian musculoskeletal physiotherapists with expertise in treating shoulder conditions. ${ }^{27}$ The intervention was directed at improving dynamic scapular control, strengthening scapular stabiliser and rotator cuff muscles, improving shoulder and thoracic posture, and increasing range of motion of thoracic extension. The intervention had five components comprising soft tissue massage, passive mobilisation of the glenohumeral joint, scapular retraining and postural taping, spinal mobilisation (to assist in improving shoulder girdle posture and spinal range of motion), and home exercises (table 1). ${ }^{26}$ We incorporated behavioural strategies, including education, goal setting, motivation, and positive reinforcement. Home exercises were done daily, except during the first week of treatment when exercises were completed twice daily (web appendix). 
Table 1|Components of active physiotherapy intervention

\begin{tabular}{|c|c|c|}
\hline Treatment component & Description & Dosage \\
\hline Soft tissue massage & Anterior and posterior shoulder tissues, in supine and side-lying positions respectively & 6 minutes each position \\
\hline Glenohumeral joint mobilisation & $\begin{array}{l}\text { Anteroposterior and inferior joint glides in supine position with shoulder at } 45^{\circ} \text { and } 90^{\circ} \\
\text { abduction respectively }\end{array}$ & $4 \times 30$ seconds each position \\
\hline Thoracic spine mobilisation (T1-8) & In prone position, using central posteroanterior technique & Grade IV on each level: 4 minutes in total \\
\hline Cervical spine mobilisation (C5-7) & In prone position using unilateral posteroanterior technique on both sides & Grade IV on each level: 4 minutes in total \\
\hline Scapular retraining & $\begin{array}{l}\text { In side-lying position, therapist passively moves shoulder through range from elevation/ } \\
\text { protraction to retraction/depression, then assisted by participant, then independently by } \\
\text { participant; isometric holds in retraction/depression }\end{array}$ & $\begin{array}{l}\text { Weeks } 1 \text { and } 2 \text { only; } 15 \text { repetitions } \times 5 \text { repetitions with } \\
10 \text { second holds }\end{array}$ \\
\hline Postural taping & $\begin{array}{l}\text { Taping of shoulders and scapula to encourage scapular retraction and depression and } \\
\text { thoracic extension }\end{array}$ & $\begin{array}{l}\text { Continuous (day and night) for two weeks; re-applied after } \\
\text { one week by therapist }\end{array}$ \\
\hline Home exercises & Supervised and done as home programme & $\begin{array}{l}\text { Home programme: twice daily in first two weeks; once a day } \\
\text { thereafter }\end{array}$ \\
\hline
\end{tabular}

After the 10 week programme, participants in the active group were instructed to maintain their daily home exercise programme for 12 weeks.

Participants in the placebo group attended the same number of treatments as did those in the active treatment group but received sham ultrasound therapy and light application of a non-therapeutic gel to the shoulder region for 10 minutes each. They received no instruction in exercise techniques and no manual therapy. We have successfully used this same placebo protocol in previous studies. ${ }^{28-30}$ During the 12 week follow-up period, placebo participants did not receive any intervention and were not instructed to do any home exercises.

\section{Outcome measures}

The same blinded assessor (EW) evaluated all participants at baseline, at 11 weeks (at the conclusion of the supervised active or placebo intervention), and at 22 weeks after randomisation. Baseline demographic information was collected, and participants rated their expectation of a beneficial effect of active physiotherapy treatment on an ordinal scale from 1 to 5 , with higher scores indicating higher expectations.

The primary outcomes were the shoulder pain and disability index (SPADI), average pain on movement assessed by a numerical rating scale, and participants' perceived global rating of change overall. The shoulder pain and disability index is a self administered, shoulder specific index consisting of 13 items divided into two subscales - pain (five items) and function (eight items)—with responses to each item recorded on a 10 point scale. ${ }^{31-33}$ We calculated a total shoulder pain and disability index score by summing the subscales and then averaging for a score out of 100 (higher scores indicate more pain/dysfunction).

We measured participants' overall assessment of average pain on movement and pain at rest in the previous week by separate 11 point numerical rating scales ( 0 to 10$)$ numbered in $1 \mathrm{~cm}$ intervals. ${ }^{34}$ The minimal clinically important difference for shoulder pain on movement measured on this scale is 1.1 units. $^{34}$ The amount of weakness, stiffness, and interference with activities of daily living over the previous week were similarly measured.
Participants' perceived global rating of change overall and in pain, strength, and stiffness (from baseline) were recorded on separate five point Likert-type scales (1=much worse, $2=$ =slightly worse, $3=$ no change, $4=$ slightly better, $5=$ much better) ${ }^{35}$ We defined a successful outcome for each a priori as "much better" on the rating scale.

We measured generic health related quality of life with the Medical Outcomes Study 36-item short form (SF-36) (eight subscales scaled from 0-100, in which a higher score represents better health, summarised into physical function and mental health scales) and the assessment of quality of life (AQoL) instrument. ${ }^{3637}$ The latter instrument comprises 15 items covering five dimensions (illness, independent living, social relationships, physical senses, and psychological wellbeing). ${ }^{3839}$ Item responses are all ordinal scales with four levels per item. Scores are scaled from -0.04 (worse than death) to 1.00 (perfect health).

We measured isometric shoulder strength of the symptomatic limb for shoulder abduction and internal and external rotation with the Nicholas Manual Muscle tester (Lafayette, USA). For abduction, participants were in supine position with the shoulder in $90^{\circ}$ of abduction and the dynamometer positioned on the lateral surface of the distal humerus. Measurements of external and internal rotation were made in sitting position with the arm by the side against a folded towel with the elbow flexed to $90^{\circ}$ and the dynamometer positioned on the distal forearm. After a demonstration and one warm-up trial, participants were asked to push as hard as they possibly could against the dynamometer for four seconds while the tester provided consistent loud verbal encouragement. The mean reading of three maximal contractions was taken. Reliability is excellent in our laboratory (12 people with rotator cuff disease tested by two examiners twice two to four days apart: intraclass correlation coefficient $(2,3)$ values $\geq 0.89$ for intra-rater reliability of each examiner and $\leq 0.90$ for inter-rater reliability ${ }^{27}$ ).

We measured participants' adherence to treatment by recording the number of physiotherapy sessions attended (out of a maximum of 10). Participants in the active group also completed a daily log book to record the number of home exercise sessions 
completed. Adverse events and the use of co-interventions in both groups were recorded in a log book. To measure the success of blinding, we asked participants to indicate which treatment they believed they had received at the 22 week assessment.

\section{Sample size}

We calculated sample size on the basis of ability to detect a 10 point difference in improvement in total shoulder pain and disability index score, previously reported to indicate a clinically important improvement (or worsening) of shoulder function. ${ }^{32}$ Applying power calculations appropriate for analysis of covariance (adjusting for baseline shoulder pain and disability index score), to detect a difference in 11 week shoulder pain and disability index score of 10 units assuming a common between participant standard deviation of 27 and a baseline to 11 week correlation of 0.45 (from our pilot study ${ }^{27}$ ), we calculated that we

Assessed for eligibility by phone $(n=1380)$

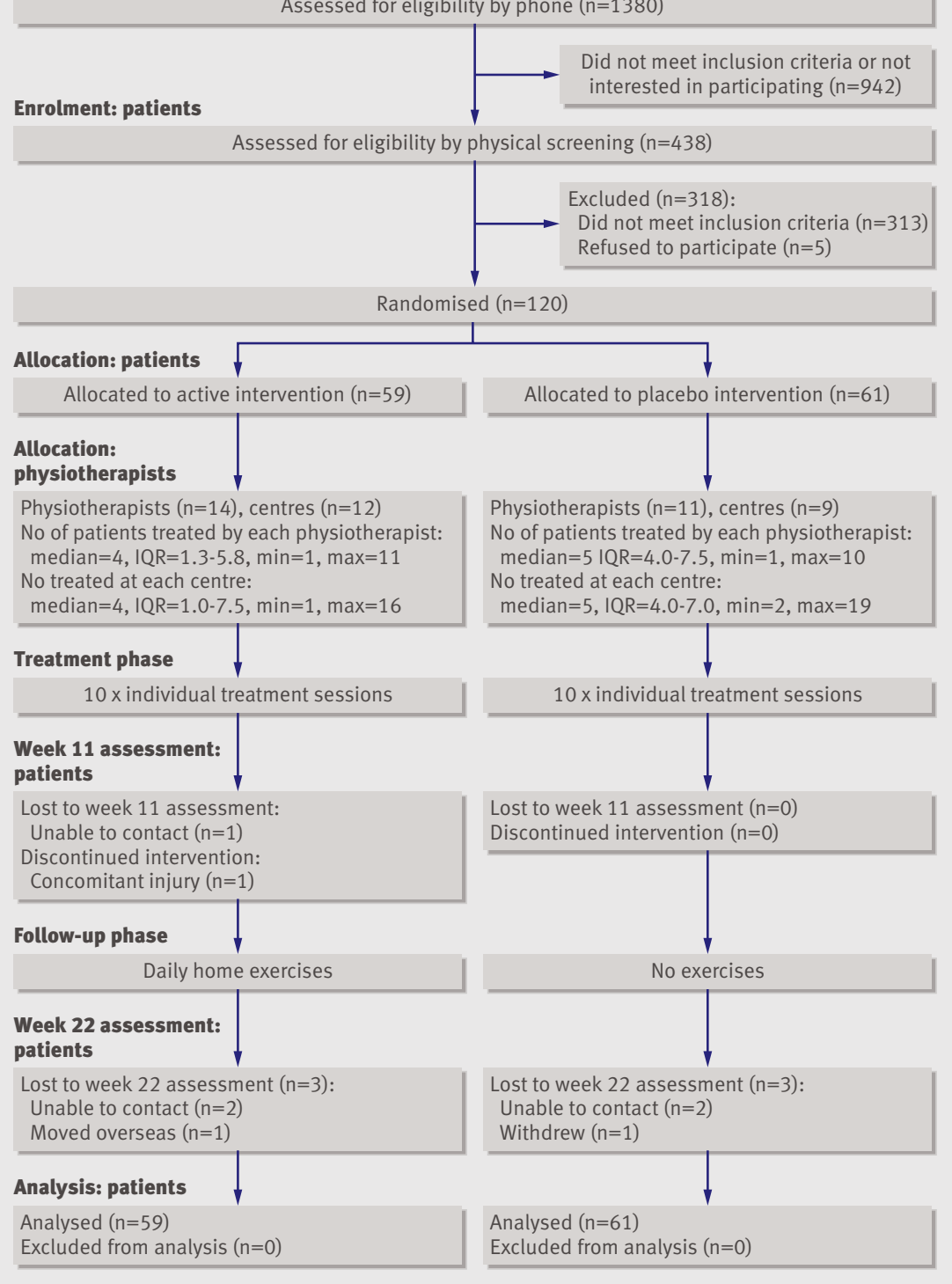

Fig 1| Flow of participants through study. IQR=interquartile range needed 91 participants per group to achieve $80 \%$ power at a two sided 5\% significance level.

As recruitment was much slower than anticipated, an independent statistician not previously associated with the trial did a blinded assessment of the between participant standard deviation and baseline to 11 week correlation after 46 patients had completed follow-up. These were more favourable than initially planned $(\mathrm{SD}=21, r=0.60)$, and we revised the total trial sample size downwards to 60 participants per arm to maintain $80 \%$ power to detect a difference of 10 units with these revised parameters.

\section{Data analysis}

We did analyses on an intention to treat principle, using all randomised participants. We replaced missing data by the last score carried forward. For outcomes measured using an essentially continuous scale, we compared differences in mean change from baseline to each time point between groups by using linear regression modelling with adjustment for baseline levels of the outcome measure. We checked model assumptions by standard diagnostic plots.

We dichotomised participants' measures of perceived global change after active or placebo treatments into successful (much better) or unsuccessful (slightly better, no change, slightly worse, and much worse) outcome. We compared the percentage of successful outcomes between groups by calculating relative risks and their 95\% confidence intervals at each time point with $\log$ binomial regression. ${ }^{40}$

We calculated an index to assess the success of blinding after treatment. ${ }^{41}$ This index takes the value one for complete blinding and zero for complete lack of blinding.

\section{RESULTS}

We recruited 120 participants (59 active, 61 placebo), and 112 (54 active, 58 placebo; 93\%) completed the 22 week trial. Figure 1 shows the flow of participants through the trial. Two participants, both from the active group, withdrew before completing the 10 week intervention. A further six participants (three active, three placebo) withdrew before the 22 week follow-up. Demographic and clinical characteristics of the eight participants who withdrew from the study did not differ from those of the 112 who remained (data not shown).

The groups were similar at baseline for demographic and clinical characteristics, although the median duration of symptoms seemed to be longer in the active group (table 2). The participants' expectation of treatment outcomes for active physiotherapy was similar in the two groups $(\mathrm{P}=0.79) ; 95 / 105(90 \%)$ participants who provided this information expected a moderate or large beneficial effect.

\section{Efficacy analysis}

Immediately after treatment (11 weeks)

Both groups showed significant improvements immediately after treatment (11 weeks). However, we found 
Table 2 Demographic and clinical characteristics of active and placebo groups. Values are numbers (percentages) unless stated otherwise

\begin{tabular}{lcc} 
Characteristic & Active $\mathrm{n}=59$ & Placebo $\mathrm{n}=61$ \\
Mean (SD) age (years) & $59.3(10.1)$ & $60.8(12.4)$ \\
\hline $\begin{array}{l}\text { Median (interquartile range) } \\
\text { duration of symptoms (months) }\end{array}$ & $24(6-54)$ & $14(6-24)$ \\
\hline Mean (SD) height (cm) & $169.0(9.1)$ & $167.5(10.8)$ \\
\hline Mean (SD) body mass (kg) & $79.5(13.5)$ & $78.9(15.9)$ \\
\hline $\begin{array}{l}\text { Mean (SD) body mass index (kg/ } \\
\left.\mathrm{m}^{2}\right)\end{array}$ & $27.8(4.4)$ & $27.9(4.8)$ \\
\hline Male sex & $34(58)$ & $30(49)$ \\
\hline Affected shoulder (right:left) & $35: 24$ & $38: 23$ \\
\hline Dominant side affected & $38(64)$ & $42(69)$ \\
\hline Previous treatment: & $29(49)$ & $35(57)$ \\
\hline Physiotherapy & $20(34)$ & $26(43)$ \\
\hline Corticosteroid injection & $10(17)$ & $15(25)$ \\
\hline Massage & $2(3)$ & $1(2)$ \\
\hline Acupuncture & $5(8)$ & $4(7)$ \\
\hline Chiropractic/osteopathy & $6(10)$ & $6(10)$ \\
\hline
\end{tabular}

no significant between group differences for the primary outcomes of changes in shoulder pain and disability index total score $(3.6,95 \%$ confidence interval -2.1 to 9.4$)$ and pain on movement $(0.7,-0.1$ to 1.5$)$ (table 3, fig 2). More participants in the active group reported an overall successful outcome (defined as "much better"), although the difference was not statistically significant: $42 \%$ of active participants and 30\% of placebo participants (relative risk 1.43, 95\% confidence interval 0.87 to 2.34 ) (table 4 ).

With regards to the secondary outcomes, the active group showed significantly greater improvements in both self reported and objective measures of strength (tables 3 and 4). We found no significant differences between treatment groups for other outcomes, including changes in health related quality of life.

\section{Twenty-two week follow-up}

The active group showed a significantly greater improvement in shoulder pain and disability index total score at 22 weeks than did the placebo group (mean between group difference $7.1,0.3$ to 13.9 ) (table 3, fig 2). However, this was not accompanied by significant differences between the active and placebo groups for change in pain on movement (table 3 , fig 2) or for the percentage of participants reporting an overall successful treatment outcome (table 4). Within group changes in the primary outcomes remained significant at the 22 week follow-up (all $\mathrm{P}<0.001)$.

Several secondary outcomes also showed benefits in favour of the active group. We saw greater improvements in shoulder pain and disability index function score, muscle strength, interference with activity, and health related quality of life as measured by the assessment of quality of life instrument (tables 3 and 4).

\section{Other analyses}

The results immediately after treatment and at followup were essentially unaltered when reanalysed comparing the active group participants who reported more than $50 \%$ adherence to the home exercise programme $(\mathrm{n}=35)$ with the placebo participants; as a completers' analysis without replacing the missing values; controlling for duration of symptoms by adding a term in the regression analysis; and using generalised estimating equations to fit population averaged models (data not shown).

\section{Adherence, adverse events, and co-interventions}

Fifty-two $(91 \%)$ of 57 participants in the active group and $57 / 61(93 \%)$ of those in the placebo group attended all 10 physiotherapy treatment sessions. The number of sessions attended by the remainder ranged from three to nine with a mean of 4.8 (SD 2.7) in the active group and from one to seven with a mean of $6.0(3.5)$ in the placebo group. Of the active group participants who completed the exercise diaries (52/57 during the intervention period and 39/55 in the follow-up period), the mean self reported completion of home exercise sessions was 57.2 (SD 15.7, 82\%) during the intervention period and $49.0(20.3,70 \%)$ during the follow-up period.

During the intervention period, 17/55 (31\%) participants in the active group reported adverse events. These comprised increased short term pain during or after the treatment session $(n=3)$, increased short term pain with the home exercises (12), and mild irritation to the tape used for postural taping (2). In the placebo group, 5/61 (8\%) reported adverse events comprising increased short term pain during or after the treatment session. During the follow-up period, adverse events were reported only by the active group (7/49, 14\%) and comprised increased short term pain with the home exercises.

Use of analgesics and non-steroidal anti-inflammatory drugs was similar in the active and placebo groups over both the intervention period (analgesics: 11/55 $(20 \%)$ active $v 14 / 61(23 \%)$ placebo; non-steroidal anti-inflammatories: 12/55 (22\%) v 13/61 (21\%); both $\mathrm{P}>0.05$ ) and the follow-up period (analgesics: 8/49 $(16 \%)$ v 8/55 (15\%); non-steroidal anti-inflammatories 6/49 (12\%) v 8/55 (15\%); both $\mathrm{P}>0.05)$.

During the intervention period, one $(2 \%)$ placebo participant received a cortisone injection into the shoulder. During the follow-up period, two (4\%) participants in the active group received a cortisone injection into the shoulder and one $(2 \%)$ participant in the placebo group received acupuncture treatment.

\section{Success of blinding}

In the active group, 32/55 (58\%) participants correctly identified their treatment group at 11 weeks compared with 21/61 (34\%) participants in the placebo group; 15 (27\%) participants in the active group were uncertain which treatment they had received compared with 27 (44\%) participants in the placebo group; and $8(15 \%)$ participants in the active group incorrectly identified their treatment group compared with 13 (21\%) participants in the placebo group. The blinding index was 0.70 (bootstrap 95\% confidence interval 0.58 to 0.82 ), 
Table $3 \mid$ Mean (SD) of groups, mean (SD) difference within groups, and mean ( $95 \% \mathrm{Cl})$ difference between groups adjusted for baseline scores for outcomes with interval data

\begin{tabular}{|c|c|c|c|c|c|c|c|c|c|c|c|c|}
\hline \multirow[b]{3}{*}{ Outcome } & \multicolumn{6}{|c|}{ Groups } & \multicolumn{4}{|c|}{ Difference within groups* } & \multicolumn{2}{|c|}{$\begin{array}{c}\text { Difference between } \\
\text { groups } \dagger\end{array}$} \\
\hline & \multicolumn{2}{|c|}{ Week 0} & \multicolumn{2}{|c|}{ Week 11} & \multicolumn{2}{|c|}{ Week 22} & \multicolumn{2}{|c|}{$\begin{array}{l}\text { Week } 0 \\
\text { to week } 11\end{array}$} & \multicolumn{2}{|c|}{$\begin{array}{c}\text { Week } 0 \\
\text { to week } 22\end{array}$} & \multirow{2}{*}{$\begin{array}{c}\text { Week } 0 \\
\text { to week } \\
11\end{array}$} & \multirow[b]{2}{*}{$\begin{array}{l}\text { Week } 0 \text { to } \\
\text { week } 22\end{array}$} \\
\hline & $\begin{array}{l}\text { Active } \\
(n=59)\end{array}$ & $\begin{array}{l}\text { Placebo } \\
(n=61)\end{array}$ & $\begin{array}{l}\text { Active } \\
(n=59)\end{array}$ & $\begin{array}{c}\text { Placebo } \\
(n=61)\end{array}$ & $\begin{array}{l}\text { Active } \\
(n=59)\end{array}$ & $\begin{array}{l}\text { Placebo } \\
(n=61)\end{array}$ & Active & Placebo & Active & Placebo & & \\
\hline SPADI total (0-100) & $\begin{array}{c}43.3 \\
(18.9)\end{array}$ & $\begin{array}{c}43.9 \\
(17.5)\end{array}$ & $\begin{array}{c}27.2 \\
(18.9)\end{array}$ & $\begin{array}{c}31.2 \\
(21.0)\end{array}$ & $\begin{array}{c}20.9 \\
(18.6)\end{array}$ & $\begin{array}{l}28.3 \\
(24.5)\end{array}$ & $\begin{array}{c}16.1 \\
(17.7)\end{array}$ & $\begin{array}{l}12.7 \\
(16.3)\end{array}$ & $\begin{array}{c}22.4 \\
(22.0)\end{array}$ & $\begin{array}{c}15.6 \\
(17.8)\end{array}$ & $\begin{array}{l}3.6(-2.1 \\
\text { to } 9.4)\end{array}$ & $\begin{array}{c}7.1(0.3 \text { to } \\
13.9)\end{array}$ \\
\hline $\begin{array}{l}\text { Pain on movement } \\
(0-10)\end{array}$ & $4.9(2.2)$ & $4.9(1.8)$ & $2.9(2.3)$ & $3.6(2.3)$ & $2.4(2.4)$ & $3.3(2.7)$ & $2.1(2.6)$ & $1.3(2.2)$ & $2.6(2.9)$ & $1.6(2.4)$ & $\begin{array}{l}0.7(-0.1 \\
\text { to } 1.5)\end{array}$ & $\begin{array}{c}0.9(-0.03 \\
\text { to } 1.7)\end{array}$ \\
\hline SPADI pain (0-100) & $\begin{array}{c}47.8 \\
(20.1)\end{array}$ & $\begin{array}{c}48.4 \\
(17.5)\end{array}$ & $\begin{array}{c}29.8 \\
(20.8)\end{array}$ & $\begin{array}{c}33.9 \\
(22.7)\end{array}$ & $\begin{array}{l}23.0 \\
(21.0)\end{array}$ & $\begin{array}{c}31.0 \\
(26.0)\end{array}$ & $\begin{array}{c}18.0 \\
(18.8)\end{array}$ & $\begin{array}{c}14.4 \\
(18.5)\end{array}$ & $\begin{array}{c}24.8 \\
(23.7)\end{array}$ & $\begin{array}{c}17.3 \\
(19.6)\end{array}$ & $\begin{array}{c}3.2(-3.2 \\
\text { to } 9.6)\end{array}$ & $\begin{array}{c}6.8(-0.7 \text { to } \\
14.3)\end{array}$ \\
\hline $\begin{array}{l}\text { SPADI function } \\
(0-100)\end{array}$ & $\begin{array}{c}33.6 \\
(20.0)\end{array}$ & $\begin{array}{l}33.8 \\
(20.2)\end{array}$ & $\begin{array}{c}20.0 \\
(16.3)\end{array}$ & $\begin{array}{c}25.1 \\
(19.3)\end{array}$ & $\begin{array}{c}14.1 \\
(14.6)\end{array}$ & $\begin{array}{c}22.2 \\
(22.8)\end{array}$ & $\begin{array}{c}13.6 \\
(17.3)\end{array}$ & $8.7(13.9)$ & $\begin{array}{l}19.6 \\
(20.7)\end{array}$ & $\begin{array}{c}11.6 \\
(16.6)\end{array}$ & $\begin{array}{l}4.7(-0.1 \\
\text { to } 9.5)\end{array}$ & $\begin{array}{c}7.6 \text { (1.8 to } \\
13.4)\end{array}$ \\
\hline Pain at rest $(0-10)$ & $2.3(1.9)$ & $2.1(1.8)$ & $1.4(1.9)$ & $1.7(1.9)$ & $1.0(2.0)$ & $1.6(2.1)$ & $1.0(2.2)$ & $0.4(2.0)$ & $1.3(2.5)$ & $0.4(2.5)$ & $\begin{array}{c}0.4(-0.2 \\
\text { to } 1.1)\end{array}$ & $\begin{array}{c}0.7(-0.1 \text { to } \\
1.4)\end{array}$ \\
\hline $\begin{array}{l}\text { Weakness on } \\
\text { movement }(0-10)\end{array}$ & $4.6(2.4)$ & $4.1(2.3)$ & $2.6(2.3)$ & $3.2(2.4)$ & $2.1(2.3)$ & $2.9(2.6)$ & $2.0(2.6)$ & $0.9(2.0)$ & $2.5(3.0)$ & $1.1(2.7)$ & $\begin{array}{l}0.8(0.05 \\
\text { to } 1.5)\end{array}$ & $\begin{array}{c}0.9(0.1 \text { to } \\
1.8)\end{array}$ \\
\hline $\begin{array}{l}\text { Stiffness on } \\
\text { movement }(0-10)\end{array}$ & $3.3(2.6)$ & $3.4(2.4)$ & $2.1(2.1)$ & $2.6(2.2)$ & $1.9(2.3)$ & $2.6(2.4)$ & $1.2(2.2)$ & $0.8(2.2)$ & $1.4(2.7)$ & $0.8(2.7)$ & $\begin{array}{l}0.4(-0.2 \\
\text { to } 1)\end{array}$ & $\begin{array}{c}0.7(-0.1 \text { to } \\
1.5)\end{array}$ \\
\hline $\begin{array}{l}\text { Interference with } \\
\text { activity }(0-10)\end{array}$ & $3.9(2.5)$ & $3.8(2.3)$ & $2.0(1.9)$ & $2.6(2.1)$ & $1.5(1.9)$ & $2.5(2.6)$ & $1.9(2.3)$ & $1.2(1.9)$ & $2.4(2.7)$ & $1.3(2.4)$ & $\begin{array}{c}0.6(-0.04 \\
\text { to } 1.2)\end{array}$ & $\begin{array}{c}0.9(0.1 \text { to } \\
1.7)\end{array}$ \\
\hline $\begin{array}{l}\text { SF-36 physical } \\
(0-100)\end{array}$ & $\begin{array}{l}49.3 \\
(23.4)\end{array}$ & $\begin{array}{c}48.9 \\
(25.0)\end{array}$ & $\begin{array}{l}61.0 \\
(28.1)\end{array}$ & $\begin{array}{l}55.0 \\
(27.5)\end{array}$ & $\begin{array}{l}60.0 \\
(27.2)\end{array}$ & $\begin{array}{l}53.5 \\
(29.1)\end{array}$ & $\begin{array}{l}11.7 \\
(26.5)\end{array}$ & $6.1(17.4)$ & $\begin{array}{l}10.8 \\
(25.0)\end{array}$ & $4.7(22.3)$ & $\begin{array}{l}5.7(-2.1 \\
\text { to } 13.6)\end{array}$ & $\begin{array}{c}6.3(-2.0 \text { to } \\
14.5)\end{array}$ \\
\hline SF-36 mental (0-100) & $\begin{array}{c}70.2 \\
(23.4)\end{array}$ & $\begin{array}{l}61.5 \\
(21.4)\end{array}$ & $\begin{array}{c}69.7 \\
(22.1)\end{array}$ & $\begin{array}{c}61.9 \\
(20.7)\end{array}$ & $\begin{array}{c}69.3 \\
(20.4)\end{array}$ & $\begin{array}{c}63.3 \\
(21.0)\end{array}$ & $\begin{array}{c}-0.6 \\
(19.3)\end{array}$ & $0.4(16.0)$ & $\begin{array}{l}-1.0 \\
(19.7)\end{array}$ & $1.8(15.8)$ & $\begin{array}{c}2.1 \\
(-3.8 \text { to } 8)\end{array}$ & $\begin{array}{c}0.6(-5.2 \text { to } \\
6.4)\end{array}$ \\
\hline AQoL (-0.4-1.0) & $0.7(0.1)$ & $0.7(0.2)$ & $0.7(0.2)$ & $0.7(0.2)$ & $0.7(0.2)$ & $0.7(0.2)$ & $0.0(0.1)$ & $0.0(0.1)$ & $0.0(0.2)$ & $0.0(0.1)$ & $\begin{array}{c}0.0(-0.04 \\
\text { to } 0.03)\end{array}$ & $\begin{array}{c}0.0(0.04 \text { to } \\
0.1)\end{array}$ \\
\hline $\begin{array}{l}\text { Abduction strength } \\
(\mathrm{kg})\end{array}$ & $7.2(5.4)$ & $6.2(3.4)$ & $8.4(4.7)$ & $6.7(3.8)$ & $8.3(3.8)$ & $6.5(3.9)$ & $1.2(3.9)$ & $0.5(2.2)$ & $1.1(4.4)$ & $0.4(2.5)$ & $\begin{array}{l}1.0(-0.1 \\
\text { to } 2)\end{array}$ & $\begin{array}{c}1.2(0.1 \text { to } \\
2.3)\end{array}$ \\
\hline $\begin{array}{l}\text { External rotation } \\
\text { strength }(\mathrm{kg})\end{array}$ & $8.1(4.5)$ & $7.1(2.6)$ & $8.3(3.9)$ & $7.2(3.0)$ & $8.4(3.6)$ & $7.0(3.0)$ & $0.2(3.8)$ & $0.1(1.4)$ & $0.3(4.3)$ & $-0.1(1.9)$ & $\begin{array}{c}0.5(-0.4 \\
\text { to } 1.4)\end{array}$ & $\begin{array}{c}0.9(-0.1 \text { to } \\
1.9)\end{array}$ \\
\hline $\begin{array}{l}\text { Internal rotation } \\
\text { strength }(\mathrm{kg})\end{array}$ & $10.9(5.5)$ & $10.2(4.5)$ & $11.7(5.2)$ & $10.1(4.3)$ & $12.2(5.3)$ & $10.2(4.6)$ & $0.9(3.1)$ & $-0.1(2.7)$ & $1.3(3.4)$ & $0.0(2.7)$ & $\begin{array}{c}1.1 \text { (0.1 to } \\
2.1)\end{array}$ & $\begin{array}{c}1.5(0.4 \text { to } \\
2.5)\end{array}$ \\
\hline
\end{tabular}

AQoL=assessment of quality of life; SF-36=Medical Outcomes Study 36-item short form; SPADI=shoulder pain and disability index.

*Positive change equals improvement, and positive values favour active group.

†Results from regression analyses adjusted for baseline scores.

interpreted as a moderate to high degree of blindedness and representing a statistically significant amount of blinding beyond that expected by chance (the value of the blinded index is 0.5 for random guessing).

\section{DISCUSSION}

This randomised, participant and single assessor blinded, placebo controlled trial evaluated the efficacy of a 10 week manual therapy and home exercise programme delivered by physiotherapists for the treatment of chronic rotator cuff disease in middle aged to older adults. Immediately after the intervention (11 weeks), the standardised active treatment generally produced similar beneficial effects on shoulder pain and function, the primary endpoints of the trial, compared with a realistic placebo treatment that controlled for therapists' contact time and the therapeutic environment. Both groups improved by amounts deemed to be clinically important, ${ }^{42}$ and more than a third of participants reported a successful treatment outcome. However, we found significant differences favouring the active group for objective and subjective measures of muscle strength. At follow-up (22 weeks), we saw greater improvements with active treatment for several outcome measures. Changes in overall pain and function measured by the shoulder pain and disability index favoured the active group, although the mean between group difference of 7.1 was slightly below the 8 to 13.2 points reported in the literature as being the minimal clinically important difference. ${ }^{42}$ Several secondary outcomes also favoured the active group, including shoulder pain and disability index function score, muscle strength, interference with activity, and quality of life.

\section{Explanation of results}

The significant improvements seen in both groups over the 22 weeks may reflect natural recovery of the rotator cuff disease. Although we did not include a third "no treatment" study arm to ascertain this, natural recovery is unlikely to explain the whole effect given the long duration of symptoms, particularly in the active group, and the moderate baseline disability of the cohort-factors that have been associated with a poorer prognosis. ${ }^{434}$ Furthermore, other clinical trials in patients with chronic rotator cuff disease found minimal changes in pain or function over similar timeframes in control groups receiving no treatment. ${ }^{1419}$ 
Table $4 \mid$ Number (percentage) of participants reporting a successful outcome ("much better") compared with those reporting an unsuccessful outcome ("slightly better," "no change," "slightly worse," or "much worse") in both groups, with relative risks

\begin{tabular}{|c|c|c|c|c|c|c|}
\hline \multirow[b]{2}{*}{ Outcome } & \multicolumn{3}{|c|}{11 weeks } & \multicolumn{3}{|c|}{22 weeks } \\
\hline & $\begin{array}{l}\text { Active } \\
(n=57)\end{array}$ & $\begin{array}{c}\text { Placebo } \\
(n=61)\end{array}$ & $\begin{array}{l}\text { Relative risk } \\
\quad(95 \% \mathrm{Cl})\end{array}$ & $\begin{array}{l}\text { Active } \\
(n=54)\end{array}$ & $\begin{array}{l}\text { Placebo } \\
(n=58)\end{array}$ & $\begin{array}{l}\text { Relative risk } \\
(95 \% \mathrm{Cl})\end{array}$ \\
\hline Global change overall & $24(42)$ & $18(30)$ & $1.43(0.87$ to 2.34$)$ & $31(57)$ & $24(41)$ & $1.39(0.94$ to 2.03$)$ \\
\hline Global change in pain & $22(39)$ & $20(33)$ & $1.18(0.72$ to 1.91$)$ & $31(57)$ & $25(43)$ & $1.33(0.92$ to 1.94$)$ \\
\hline Global change in strength & $19(33)$ & $7(11)$ & 2.90 (1.32 to 6.39) & $22(41)$ & $14(24)$ & $1.69(0.97$ to 2.95$)$ \\
\hline Global change in stiffness & $20(35)$ & $13(21)$ & 1.65 (0.91 to 2.99$)$ & $25(46)$ & $18(31)$ & $1.49(0.92$ to 2.41$)$ \\
\hline
\end{tabular}

Improvement in our cohort may also have arisen from the statistical phenomenon of regression to the mean. ${ }^{45}$ This refers to the tendency for extreme symptoms at baseline to return to a more typical state at final assessment. Symptoms associated with chronic rotator cuff disease fluctuate over time, and patients often seek medical care or enrol in research when the symptoms are at their worst. Furthermore, we included patients only if their pain was worse than a specific threshold level. The next change in symptoms is thus more likely to be an improvement. ${ }^{46}$ However, we accounted for regression to the mean in our statistical analyses by adjusting for the baseline value of each variable. ${ }^{47}$

In addition to spontaneous improvement, another factor contributing to the total treatment effect is the placebo effect. ${ }^{48}$ A recent meta-analysis showed that for active treatment of chronic pain conditions (not specifically of the shoulder), spontaneous recovery contributes around $10 \%$ and placebo effects around $30 \%{ }^{46}$ Placebo effects have also been found to be greatest for non-drug interventions and for patient reported outcomes, particularly pain. ${ }^{49}$ Other factors contributing to the placebo effect in our trial include blinding of participants, a treatment protocol involving considerable interaction with the therapist (10 individual sessions), and the fact that most $(90 \%)$ participants expected to gain a moderate to large benefit from active treatment (given that positive expectations are known to be associated with improved outcomes $\left.{ }^{50}\right)$. Interestingly, the $33 \%$ reduction in pain reported by the placebo group is consistent with the $38 \%$ reduction found in the placebo group of our previous study in patients with osteoarthritis of the knee, in which we used an identical placebo treatment and pain measure. ${ }^{28}$

Our primary outcome measures included an assessment of pain but did not include an assessment of function in isolation. The results of both the primary and secondary outcomes suggest that active treatment did not substantially affect pain compared with placebo. However, evidence from the secondary outcome of shoulder pain and disability index function score suggests that shoulder function was improved to a significantly greater extent with active treatment. Given that our primary outcome of shoulder pain and disability index total score includes both the pain and function subscales, the benefits of active treatment on function could have been masked at the 11 week time point and attenuated at the 22 week time point, when a significant treatment group effect was found for shoulder pain and disability index total score. Although the mean between group difference at the latter time point (7.1 units) was slightly below the minimal clinically important difference we used when designing the study, the 95\% confidence interval includes the 10 unit threshold. This, together with the fact that the active treatment also led to significantly greater improvements in many secondary outcomes, indicates that manual therapy and home exercise may be beneficial particularly over time.

\section{Aspects of active and placebo interventions}

Some debate exists in the literature about whether the use of a placebo treatment as a comparator for complex interventions such as physiotherapy is appropriate. ${ }^{48}$ The direct and indirect (placebo) effects of the therapy have been argued to be unlikely to be distinct and divisible, and elements that may be categorised as indirect effects in drug trials may in fact be integral to many non-drug interventions. Hence, using a placebo controlled trial design to test an intervention such as physiotherapy can mean that the differences between the groups substantially underestimate the total effects
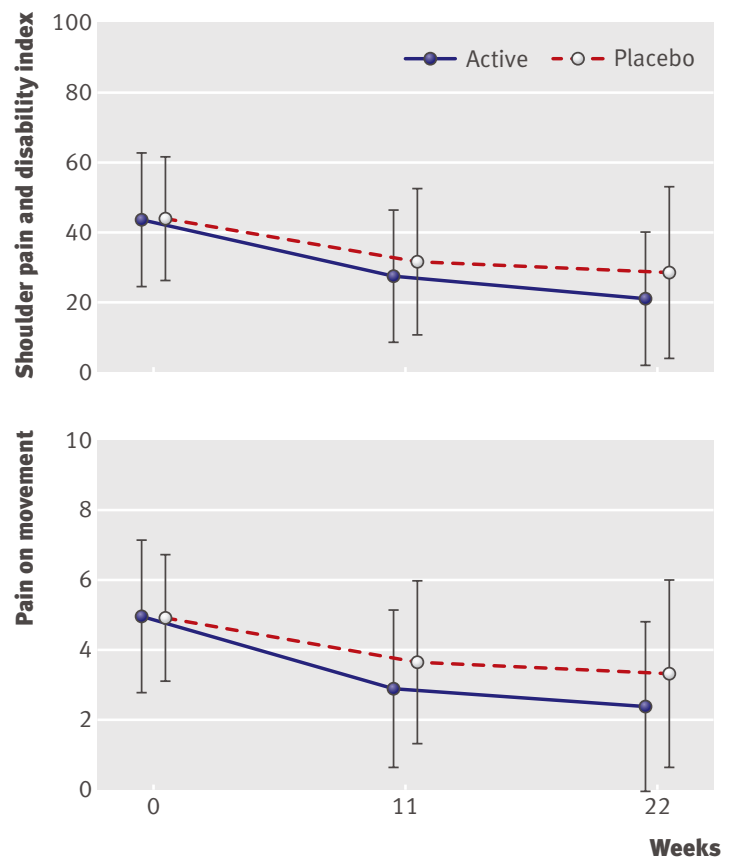

$\overline{\text { Fig } 2}$ Mean (SD) shoulder pain and disability index (SPADI) and pain on movement for active and placebo groups at baseline, 11 weeks, and 22 weeks 
of treatment. This can lead to false negative results and erroneous conclusions about efficacy. We would have found significant beneficial effects of treatment if we had compared the active treatment with a no treatment control group as other studies in this area have done. $^{21-23}$

Several aspects of the active intervention warrant consideration. Firstly, our standardised programme could be argued to have failed to adequately treat the specific physical impairments that patients presented with and that relate to shoulder pain and dysfunction. We noted significant improvements in isometric strength of $12-15 \%$ for the shoulder abductor and internal rotator muscles, as well as self reported strength gains, suggesting some effect. Whether the active treatment also successfully tackled other physical factors such as dynamic scapular control and thoracic posture is unknown, as these were not measured. Limited research shows that manual therapy techniques and exercises similar to those used in our study can alter shoulder and trunk biomechanics. ${ }^{51}$

Secondly, several participants failed to complete more than half of the prescribed home exercises, particularly during the unsupervised follow-up period. Problems with adherence to exercise programmes are common and reinforce the need to better incorporate strategies to enhance adherence, particularly when formal supervision by therapists ceases. However, our results were unaltered when we reanalysed the data excluding participants who failed to complete more than $50 \%$ of the home exercises, suggesting that levels of adherence in this study did not unduly influence the outcome.

Thirdly, to ensure a consistent approach and allow replication, we chose to evaluate a standardised treatment programme based on common elements identified from our survey and from the literature. It does not reflect the practice of every clinician involved in the conservative management of rotator cuff disease, and our results cannot necessarily be generalised to other manual therapy and exercise programmes given differences in type and dosage. Furthermore, as our treatment was standardised it may have been ineffective or even inappropriate for some patients, thus worsening symptoms and attenuating the treatment effects in the active group. However, a similar proportion of participants in both groups reported that they were worse after treatment $(9 / 57(16 \%)$ in the active group; $7 / 61$ $(12 \%)$ in the placebo group), suggesting that this was not the case. Further research is needed to evaluate the efficacy of other physiotherapy protocols for chronic rotator cuff disease and to assess treatment that is tailored to individual patients, as occurs in clinical practice. ${ }^{52}$

Like most other trials of rotator cuff disease to date, we chose to include participants on the basis of clinical features alone. Rotator cuff disease is known to most commonly affect the supraspinatus tendon, ${ }^{10}$ but we cannot exclude the possibility that differences existed in the underlying structural abnormalities within our study population, although these were likely to be equally distributed between the treatment groups. Imaging techniques such as magnetic resonance imaging or ultrasonography may further improve diagnostic accuracy, ${ }^{53}$ but these still lack sensitivity for certain pathological features, ${ }^{5455}$ are costly, and are generally not used in the primary care setting. Furthermore, physiotherapy treatment is not directed at the specific pathology but at the movement dysfunction and potential underlying mechanisms such as altered muscle function, tight structures, and poor scapular and spinal posture that have been reported in patients with rotator cuff disease. ${ }^{51}$ Whether better outcomes with physiotherapy could be gained if subgroups of rotator cuff disease could be defined and specifically treated is not known.

\section{Comparison with previous studies}

A limited number of randomised controlled trials of physiotherapy modalities for chronic rotator cuff disease have been done, and none has tested a combined intervention of manual therapy and exercise against a placebo control to allow a direct comparison with our results. The only study to use a placebo control treatment (detuned laser twice weekly for six weeks) evaluated a three to six month exercise programme supervised by physiotherapists. ${ }^{56}$ This trial found that exercise resulted in a significant $66 \%$ reduction in pain (measured on the Neer score), which is slightly higher than the $43 \%$ reduction in pain with our active treatment. However, unlike our study, the researchers noted little improvement in their placebo group, rendering their between group differences significant. Whether blinding of participants was successfully achieved was not stated. Other studies have compared exercise with arthroscopic surgery and shown similar beneficial outcomes in patients with rotator cuff disease. ${ }^{56-58}$ The limited studies evaluating exercise combined with manual therapy have used an exercise only group as the comparator. ${ }^{59-61}$ These have found that the effects of exercise on both pain and function are augmented with manual therapy, providing a rationale for evaluating a combined intervention.

\section{Strengths and weaknesses}

The strengths of our study include the rigorous study design, adequate statistical power, excellent retention of participants, inclusion of a placebo control, and the use of several therapists and a variety of recruitment sources to increase the external validity of the results. Our study has some limitations. Therapists were not blinded to treatment group, which is unavoidable in a trial of this nature. However, their interaction with patients was standardised and any bias due to nonblinding of therapists would probably favour the active group, which was not particularly evident in the outcomes. Participants were blinded, but given the difficulty in designing a credible placebo for physiotherapy interventions, blinding may not have been as complete as can be achieved in a drug trial in which an identical placebo pill can be administered. However, formal testing of the success of blinding indicated that we achieved a moderate to high degree of blindedness, representing a statistically significant amount of 


\section{WHAT IS ALREADY KNOWN ON THIS TOPIC}

Rotator cuff disease is a common shoulder condition causing pain and loss of function

Manual therapy techniques and exercise programmes are often used in the management of rotator cuff disease, yet little conclusive evidence supports or refutes their efficacy

\section{WHAT THIS STUDY ADDS}

Immediate beneficial effects of a standardised manual therapy and home exercise programme were comparable to those of a realistic placebo treatment in middle aged to older adults with chronic rotator cuff disease

Benefits of manual therapy and exercise may accrue over time and may be of more value for improving shoulder function than pain

blinding beyond that expected by chance. Our sample size was reduced after a blinded interim analysis but gave sufficient power $(80 \%)$ to detect clinically meaningful differences in the primary outcome. We replaced missing values by using the last observation carried forward method that was commonly used at the time the study was being planned but has fallen out of favour more recently ${ }^{62}$ This method is unlikely to have influenced the results, given the small dropout rate (8/ 120 participants, $7 \%$ ) and the fact that we found similar outcomes when we used generalised estimating equations to fit population averaged models to the known scores.

\section{Conclusions and practice implications}

Our study showed that the particular manual therapy and home exercise programme tested conferred no additional benefit immediately after treatment compared with a realistic placebo in middle aged to older adults with chronic rotator cuff disease. However, given evidence of significantly greater improvements with active treatment at follow-up in one of the primary outcomes and in several secondary outcomes, the benefits of manual therapy and exercise may accrue over time. Physiotherapy interventions may also be of more value for improving shoulder function than pain per se in this population. Clinicians should thus establish whether the patient's primary presenting problem is pain, impaired function, or both. If pain is a major factor, then other treatments that reduce pain, such as corticosteroid injections, may be more appropriate. If both pain and loss of function are factors, then drugs or other pain relieving treatments may be needed in combination with manual therapy and exercise to adequately treat all facets. To facilitate adherence, clinicians should advise patients that the effects of manual therapy and exercise are not necessarily immediate but may take several months before they are evident. Further research is needed to evaluate the effectiveness of different physiotherapy treatment regimens and whether the combination of drug treatment with physiotherapy leads to greater benefits in people with mild to moderate chronic rotator cuff disease.

We acknowledge the support and contribution of the Physiotherapy Department of the Royal Melbourne Hospital, particularly Geraldine Millard and Lauren Andrew, as well as the project physiotherapists Richard Bohen, Tanja Farmer, Marie-Louise Francken, Nigel Friend, Jean
Leaf, Arthur Lee, Stephen Maloney, Christine Roberts, Paul Visentini, Jennifer Maciel, Richard Burton, Jane Burman, Debbie Virtue, and Simon Wilson. Ben Metcalf also assisted with data analysis and preparation of the manuscript.

Contributors: $\mathrm{KB}, \mathrm{SC}$, and RB conceived and designed the trial protocol. $\mathrm{KB}, \mathrm{RB}, \mathrm{SG}, \mathrm{SC}, \mathrm{AH}$, and $\mathrm{AF}$ procured the project funding. SC and SG designed the physiotherapy intervention. AF designed and coordinated the statistical analysis and generated the randomisation table. MS did the analyses. EW was the project manager and blinded assessor. KB drafted the manuscript, and $\mathrm{RB}, \mathrm{EW}, \mathrm{SC}, \mathrm{SG}, \mathrm{AH}, \mathrm{MS}$, and $\mathrm{AF}$ contributed to the manuscript. All authors read and approved the final manuscript. KB is the guarantor

Funding: KB is funded in part by an Australian Research Council future fellowship. RB is funded in part by an Australian National Health and Medical Research Council practitioner fellowship. This work was funded by the National Health and Medical Research Council (project grant \#299840). Pilot funds were provided by ANZ Trustees, Department of Physiotherapy and Victor Hurley Grant Royal Melbourne Hospital and the School of Physiotherapy, University of Melbourne. The study sponsors had no role in the design; in the collection, analysis, and interpretation of the data; or in the writing of the article and the decision to submit it for publication.

Competing interests: All authors have completed the Unified Competing Interest form at www.icmje.org/coi_disclosure.pdf (available on request from the corresponding author) and declare (1) RB and KB are partly supported by fellowships from the National Health and Medical Research Council and the Australian Research Council respectively. None of the other authors have financial support for the submitted work from anyone other than their employer; (2) No financial relationships with commercial entities that might have an interest in the submitted work; (3) No spouses, partners, or children with relationships with commercial entities that might have an interest in the submitted work; (4) No non-financial interests that may be relevant to the submitted work.

Ethical approval: The Royal Melbourne Hospital Human Research Ethics Committee approved the study (project \#2001.115), and all participants gave written informed consent.

Data sharing: Physiotherapy protocols, technical appendix, statistical code, and dataset are available from the corresponding author at k.bennell@unimelb.edu.au.

Chard MD, Hazleman R, Hazleman BL, King RH, Reiss BB. Shoulder disorders in the elderly: a community survey. Arthritis Rheum 1991;34:766-9.

2 Roquelaure Y, Ha C, Leclerc A, Touranchet A, Sauteron M, Melchior M, et al. Epidemiologic surveillance of upper-extremity musculoskeletal disorders in the working population. Arthritis Rheum 2006;55:765-78.

3 Badley EM, Tennant A. Changing profile of joint disorders with age: findings from a postal survey of the population of Calderdale, West Yorkshire, United Kingdom. Ann Rheum Dis 1992;51:366-71.

4 Smith KL, Harryman DT 2nd, Antoniou J, Campbell B, Sidles JA, Matsen FA 3rd. A prospective, multipractice study of shoulder function and health status in patients with documented rotator cuff tears. J Shoulder Elbow Surg 2000;9:395-402.

5 Bridges-Webb C, Britt H, Miles D, Neary S, Charles J. Morbidity and treatment in general practice in Australia 1990-91. Med J Aust 1992;157:1-56S.

6 Peters D, Davies P, Pietroni P. Musculoskeletal clinic in genera practice: study of one year's referrals. Br J Gen Pract 1994;44:25-9.

7 Bartolozzi A, Andreychik D, Ahmad S. Determinants of outcome in the treatment of rotator cuff disease. Clin Orthop Relat Res 1994;308:90-7.

8 Gartsman GM, Brinker MR, Khan M, Karahan M. Self-assessment of general health status in patients with five common shoulder conditions. J Shoulder Elbow Surg 1998;7:228-37.

9 Roquelaure Y, Mariel J, Fanello S, Boissiere JC, Chiron H, Dano C, et al. Active epidemiological surveillance of musculoskeletal disorders in a shoe factory. Occup Environ Med 2002;59:452-8.

10 Ostor AJ, Richards CA, Prevost AT, Speed CA, Hazleman BL. Diagnosis and relation to general health of shoulder disorders presenting to primary care. Rheumatology (Oxford) 2005;44:800-5.

11 Buchbinder R, Goel V, Bombardier C, Hogg-Johnson S. Classification systems of soft tissue disorders of the neck and upper limb: do they satisfy methodological guidelines? J Clin Epidemiol 1996;49:141-9.

12 Ekeberg OM, Bautz-Holter E, Tveita EK, Juel NG, Kvalheim S, Brox JI. Subacromial ultrasound guided or systemic steroid injection for rotator cuff disease: randomised double blind study. BMJ 2009;338:a3112.

13 Green S, Buchbinder R, Hetrick S. Physiotherapy interventions for shoulder pain. Cochrane Database Syst Rev 2003;2:CD004258 
14 Lombardi I Jr, Magri AG, Fleury AM, Da Silva AC, Natour J. Progressive resistance training in patients with shoulder impingement syndrome: a randomized controlled trial. Arthritis Rheum 2008;59:615-22.

15 Glazier RH, Dalby DM, Badley EM, Hawker GA, Bell MJ, Buchbinder R, et al. Management of common musculoskeletal problems: a survey of Ontario primary care physicians. CMAI 1998;158:1037-40.

16 Michener LA, McClure PW, Karduna AR. Anatomical and biomechanical mechanisms of subacromial impingement syndrome. Clin Biomech 2003;18:369-79.

17 Dickens VA. Role of physiotherapy in the treatment of subacromial impingement syndrome: a prospective study. Physiotherapy 2005;91:159-64.

18 Haahr JP, Ostergaard S, Dalsgaard J, Norup K, Frost P, Lausen S, et al. Exercises versus arthroscopic decompression in patients with subacromial impingement: a randomised, controlled study in 90 cases with a one year follow up. Ann Rheum Dis 2005;64:760-4.

19 Ludewig PM, Borstad JD. Effects of a home exercise programme on shoulder pain and functional status in construction workers. Occup Environ Med 2003;60:841-9.

20 Walther M, Werner A, Stahlschmidt T, Woelfel R, Gohlke F. The subacromial impingement syndrome of the shoulder treated by conventional physiotherapy, self-training, and a shoulder brace: results of a prospective, randomized study. J Shoulder Elbow Surg 2004;13:417-23.

21 Faber E, Kuiper JI, Burdorf A, Miedema HS, Verhaar JA. Treatment of impingement syndrome: a systematic review of the effects on functional limitations and return to work. J Occup Rehabil 2006;16:7-25

22 Kuhn JE. Exercise in the treatment of rotator cuff impingement: a systematic review and a synthesized evidence-based rehabilitation protocol. J Shoulder Elbow Surg 2009;18:138-60.

23 Trampas A, Kitisios A. Exercise and manual therapy for the treatment of impingement syndrome of the shoulder: a systematic review. Phys Ther Rev 2006;11:125-42.

24 Hawkins RJ, Kennedy JC. Impingement syndrome in athletes. Am J Sports Med 1980;8:151-8.

25 Maitland GD. Vertebral manipulation. Butterworth-Heinemann, 2001.

26 Bennell K, Coburn S, Wee E, Green S, Harris A, Forbes A, et al. Efficacy and cost-effectiveness of a physiotherapy program for chronic rotator cuff pathology: a protocol for a randomised, double-blind, placebocontrolled trial. BMC Musculoskelet Disord 2007;8:86.

27 Coburn S. Efficacy of physiotherapy for chronic rotator cuff pathology: a randomised, double-blind, placebo controlled pilot trial [masters of physiotherapy thesis]. University of Melbourne, 2008.

28 Bennell K, Hinman R, Metcalf B, Buchbinder R, McConnell J, McColl G, et al. Efficacy of physiotherapy management of knee joint osteoarthritis: a randomised double-blind placebo-controlled trial. Ann Rheum Dis 2005;64:906-12.

29 Buchbinder R, Youd JM, Green S, Stein A, Forbes A, Harris A, et al. Efficacy and cost-effectiveness of physiotherapy following glenohumeral joint distension for adhesive capsulitis: a randomized trial. Arthritis Rheum 2007;57:1027-37.

30 Crossley K, Bennell K, Green S, Cowan S, McConnell J. Physical therapy for patellofemoral pain: a randomized, double-blinded, placebo-controlled trial. Am J Sports Med 2002;30:857-65.

31 Heald SL, Riddle DL, Lamb RL. The shoulder pain and disability index the construct validity and responsiveness of a region-specific disability measure. Phys Ther 1997;77:1079-89.

32 Williams JW Jr, Holleman DR Jr, Simel DL. Measuring shoulder function with the shoulder pain and disability index. J Rheumatol 1995;22:727-32.

33 Roach KE, Budiman-Mak E, Songsiridej N, Lertratanakul Y. Development of a shoulder pain and disability index. Arthritis Care Res 1991;4:143-9.

34 Mintken PE, Glynn P, Cleland JA. Psychometric properties of the shortened disabilities of the arm, shoulder, and hand questionnaire (QuickDASH) and numeric pain rating scale in patients with shoulder pain. J Shoulder Elbow Surg 2009;18:920-6.

35 Ten Klooster PM, Drossaers-Bakker KW, Taal E, van de Laar MA. Patient-perceived satisfactory improvement (PPSI): interpreting meaningful change in pain from the patient's perspective. Pain 2006;121:151-7.

36 Walters SJ, Munro JF, Brazier JE. Using the SF-36 with older adults: a cross-sectional community-based survey. Age Ageing 2001;30:337-43.

37 Hawthorne G, Osborne R. Population norms and meaningful differences for the assessment of quality of life (AQoL) measure. Aus N ZJ Public Health 2005;29:136-42.

38 Osborne RH, Hawthorne G, Lew EA, Gray LC. Quality of life assessment in the community-dwelling elderly: validation of the assessment of quality of life (AQoL) instrument and comparison with the SF-36. J Clin Epidemiol 2003;56:138-47.
39 Whitfield K, Buchbinder R, Segal L, Osborne RH. Parsimonious and efficient assessment of health-related quality of life in osteoarthritis research: validation of the assessment of quality of life (AQoL) instrument. Health Qual Life Outcomes 2006;4:19.

40 McNutt LA, Wu C, Xue X, Hafner JP. Estimating the relative risk in cohort studies and clinical trials of common outcomes. Am J Epidemiol 2003;157:940-3.

41 James KE, Bloch DA, Lee KK, Kraemer HC, Fuller RK. An index for assessing blindness in a multi-centre clinical trial: disulfiram for alcohol cessation-a VA cooperative study. Stat Med 1996;15:1421-34

42 Roy JS, MacDermid JC, Woodhouse LJ. Measuring shoulder function: a systematic review of four questionnaires. Arthritis Rheum 2009;61:623-32.

43 Kuijpers T, van der Windt DA, van der Heijden GJ, Bouter LM. Systematic review of prognostic cohort studies on shoulder disorders. Pain 2004;109:420-31.

44 Thomas E, van der Windt DA, Hay EM, Smidt N, Dziedzic K, Bouter LM, et al. Two pragmatic trials of treatment for shoulder disorders in primary care: generalisability, course, and prognostic indicators. Ann Rheum Dis 2005;64:1056-61.

45 Morton V, Torgerson DJ. Regression to the mean: treatment effect without the intervention. J Eval Clin Pract 2005;11:59-65.

46 Krogsboll LT, Hrobjartsson A, Gotzsche PC. Spontaneous improvement in randomised clinical trials: meta-analysis of threearmed trials comparing no treatment, placebo and active intervention. BMC Med Res Methodol 2009;9:1.

47 Barnett AG, van der Pols JC, Dobson AJ. Regression to the mean: what it is and how to deal with it. Int J Epidemiol 2005;34:215-20.

48 Paterson C, Dieppe P. Characteristic and incidental (placebo) effects in complex interventions such as acupuncture. BMJ 2005;330:1202-5.

49 Hrobjartsson A, Gotzsche PC. Is the placebo powerless? An analysis of clinical trials comparing placebo with no treatment. $N$ Engl J Med 2001;344:1594-602

50 Crow R, Gage H, Hampson S, Hart J, Kimber A, Thomas H. The role of expectancies in the placebo effect and their use in the delivery of health care: a systematic review. Health Technol Assess 1999;3:1-96.

51 Ludewig PM, Reynolds JF. The association of scapular kinematics and glenohumeral joint pathologies. J Orthop Sports Phys Ther 2009;39:90-104.

52 May S, Greasley A, Reeve S, Withers S. Expert therapists use specific clinical reasoning processes in the assessment and management of patients with shoulder pain: a qualitative study. Aust J Physiother 2008;54:261-6.

53 Cullen D, Breidahl W, Janes G. Diagnostic accuracy of shoulder ultrasound performed by a single operator. Australas Radiol 2007;51:226-9.

54 Cothran RL Jr. Imaging in evaluating the rotator cuff. J Surg Orthop Adv 2006;15:132-9.

55 Moosmayer S, Heir S, Smith HJ. Sonography of the rotator cuff in painful shoulders performed without knowledge of clinical information: results from 58 sonographic examinations with surgical correlation. J Clin Ultrasound 2007;35:20-6.

56 Brox II, Staff PH, Ljunggren AE, Brevik JI. Arthroscopic surgery compared with supervised exercises in patients with rotator cuff disease (stage II impingement syndrome). BMJ 1993;307:899-903.

57 Haahr IP, Ostergaard S, Dalsgaard I, Norup K, Frost P, Lausen S, et al. Exercises versus arthroscopic decompression in patients with subacromial impingement: a randomised, controlled study in 90 cases with a one year follow up. Ann Rheum Dis 2005;64:760-4.

58 Ketola S, Lehtinen J, Arnala I, Nissinen M, Westenius H, Sintonen H, et al. Does arthroscopic acromioplasty provide any additional value in the treatment of shoulder impingement syndrome? A two-year randomised controlled trial. J Bone Joint Surg Br 2009;91:1326-34.

59 Bang MD, Deyle GD. Comparison of supervised exercise with and without manual physical therapy for patients with shoulder impingement syndrome. J Orthopaed Sports Phys Ther 2000;30:126-37.

60 Conroy DE, Hayes KW. The effect of joint mobilization as a component of comprehensive treatment for primary shoulde impingement syndrome. J Orthopaed Sports Phys Ther 1998;28:3-14

61 Senbursa G, Baltaci G, Atay A. Comparison of conservative treatment with and without manual physical therapy for patients with shoulder impingement syndrome: a prospective, randomized clinical trial. Knee Surg Sports Traumatol Arthrosc 2007;15:915-21.

62 Sterne JA, White IR, Carlin JB, Spratt M, Royston P, Kenward MG, et al. Multiple imputation for missing data in epidemiological and clinical research: potential and pitfalls. BMJ 2009;338:b2393.

Accepted: 25 March 2010 